\title{
Thermoelectric Oxide Ceramics Outperforming Single Crystals Enabled By Dopants Segregations
}

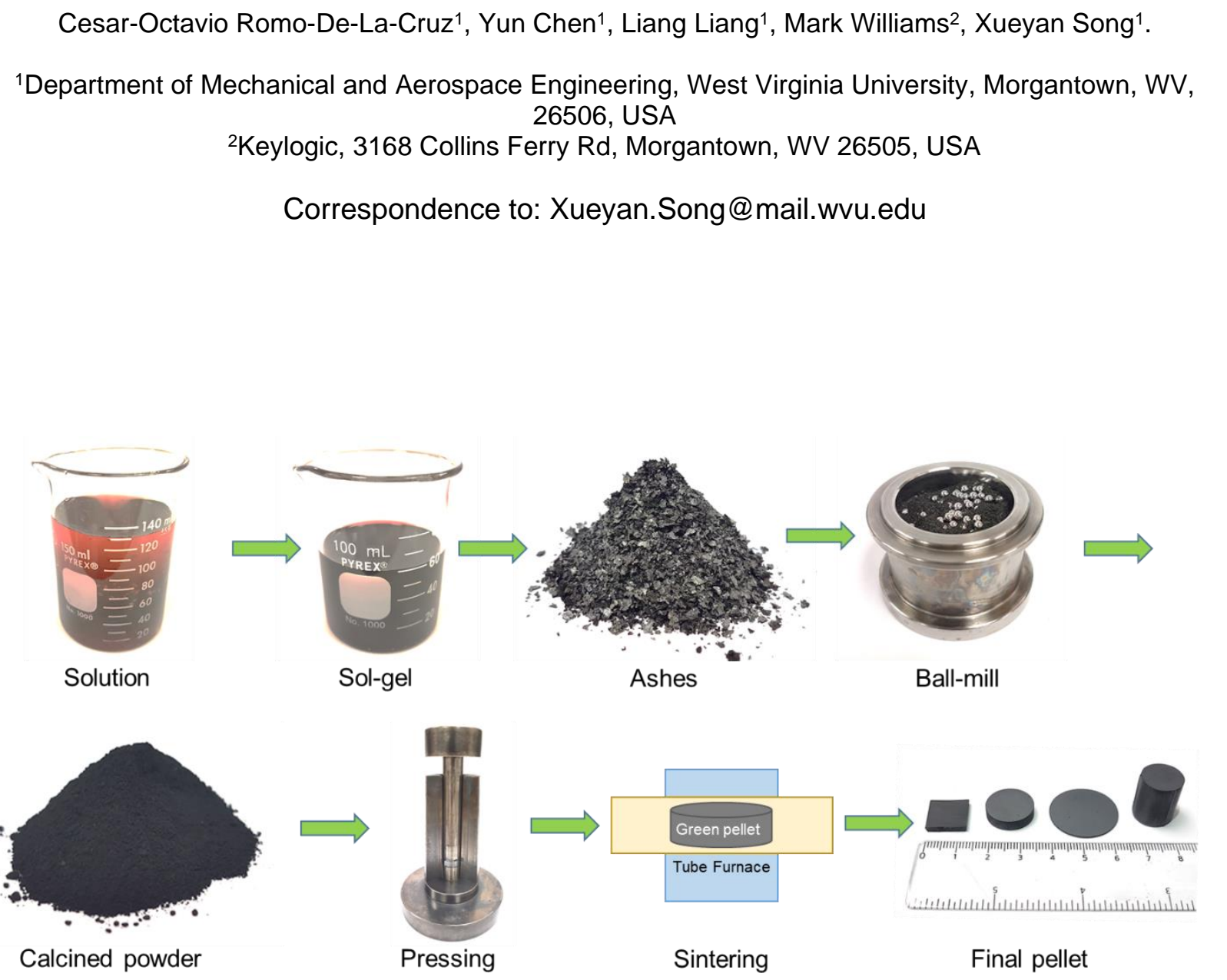

Figure S1: Procedure of fabrication polycrystalline ceramics pellets with the precursor powders made from the conventional chemical sol-gel route. 

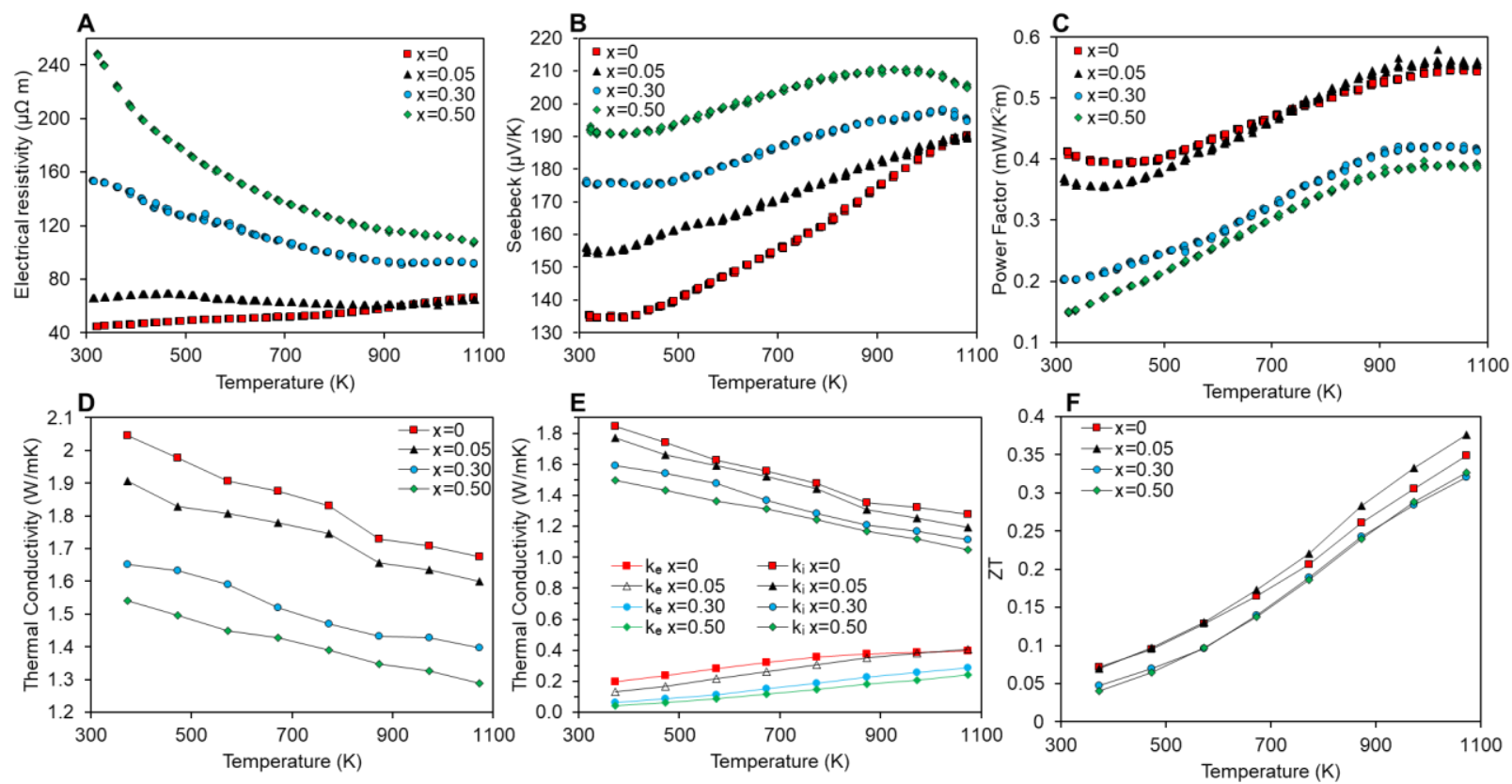

Figure S2: Temperature dependence of $\mathrm{Ca}_{3-x} \mathrm{~Tb}_{x} \mathrm{CO}_{4} \mathrm{O}_{9+\delta}$ samples: (A) electrical resistivity $(\rho-T)$, (B) Seebeck coefficient $(S-T)$, and $(C)$ electrical power factor $\left(S^{2} / \rho-T\right)$; and $(D)$ total thermal conductivity $(\kappa-T),(E)$ electronic contribution $\left(\kappa_{e}\right)$ and lattice contribution $\left(\kappa_{i}\right)$, and $(F)$ thermoelectric figure of merit $(Z T)$.
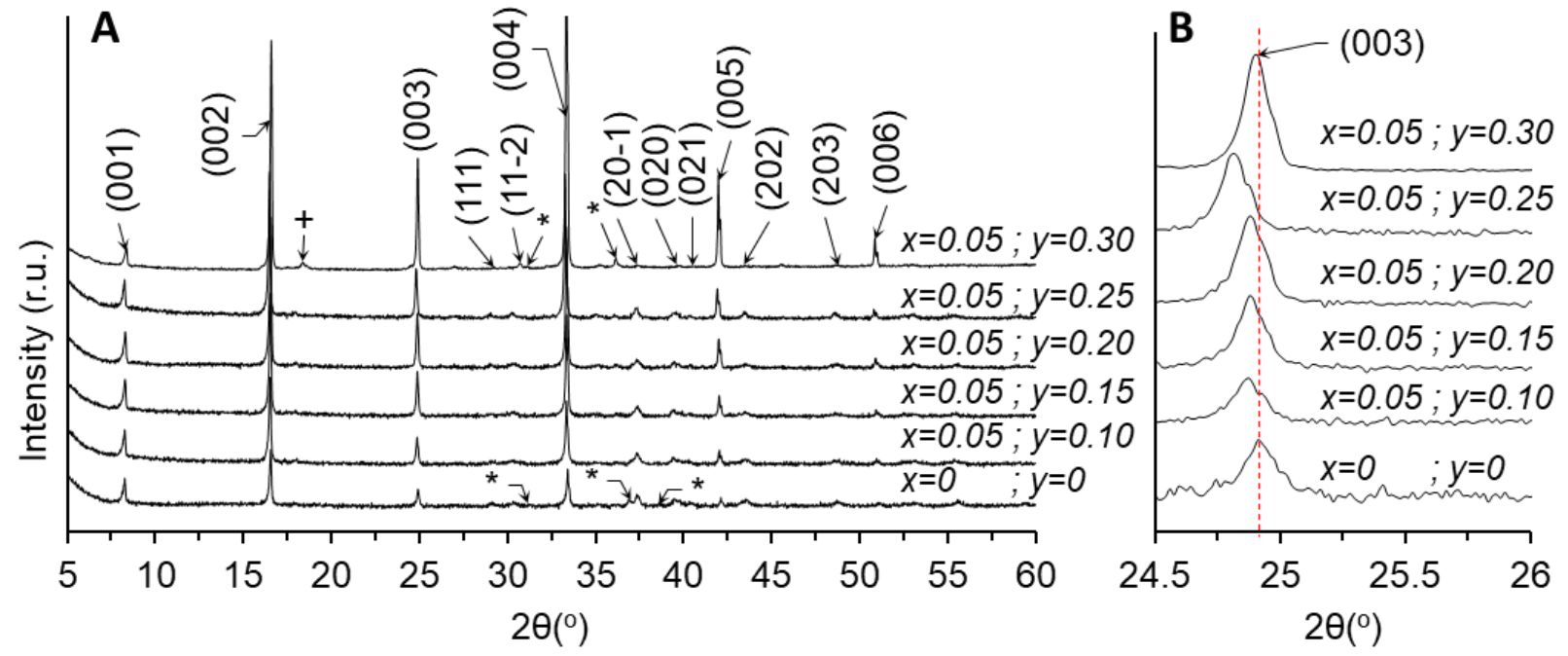

Figure S3: A: XRD powder diffraction patterns for the undoped $\mathrm{Ca}_{3} \mathrm{Co}_{4} \mathrm{O}_{9+\delta}$ and the doped $\mathrm{Ca}_{2.95} \mathrm{~Tb}_{0.05} \mathrm{Co}_{4} \mathrm{O}_{9+\delta} \mathrm{Bi}_{y}(y=0.10,0.15,0.20,0.25$, and 0.30$)$ samples. In all samples, monoclinic $\mathrm{Ca}_{3} \mathrm{Co}_{4} \mathrm{O}_{9+\delta}$ was observed. Nonetheless, a minor amount of $\mathrm{CO}_{3} \mathrm{O}_{4}$ and $\mathrm{Ca}_{3} \mathrm{CO}_{2} \mathrm{O}_{6}$ was observed as indicated by the marked peaks * and ${ }^{+}$, respectively. B: (003) diffraction peak showing the shift to a lower Bragg's angle. Red line correspond to position of peak in the undoped $\mathrm{Ca}_{3} \mathrm{Co}_{4} \mathrm{O}_{9+\delta}$. 

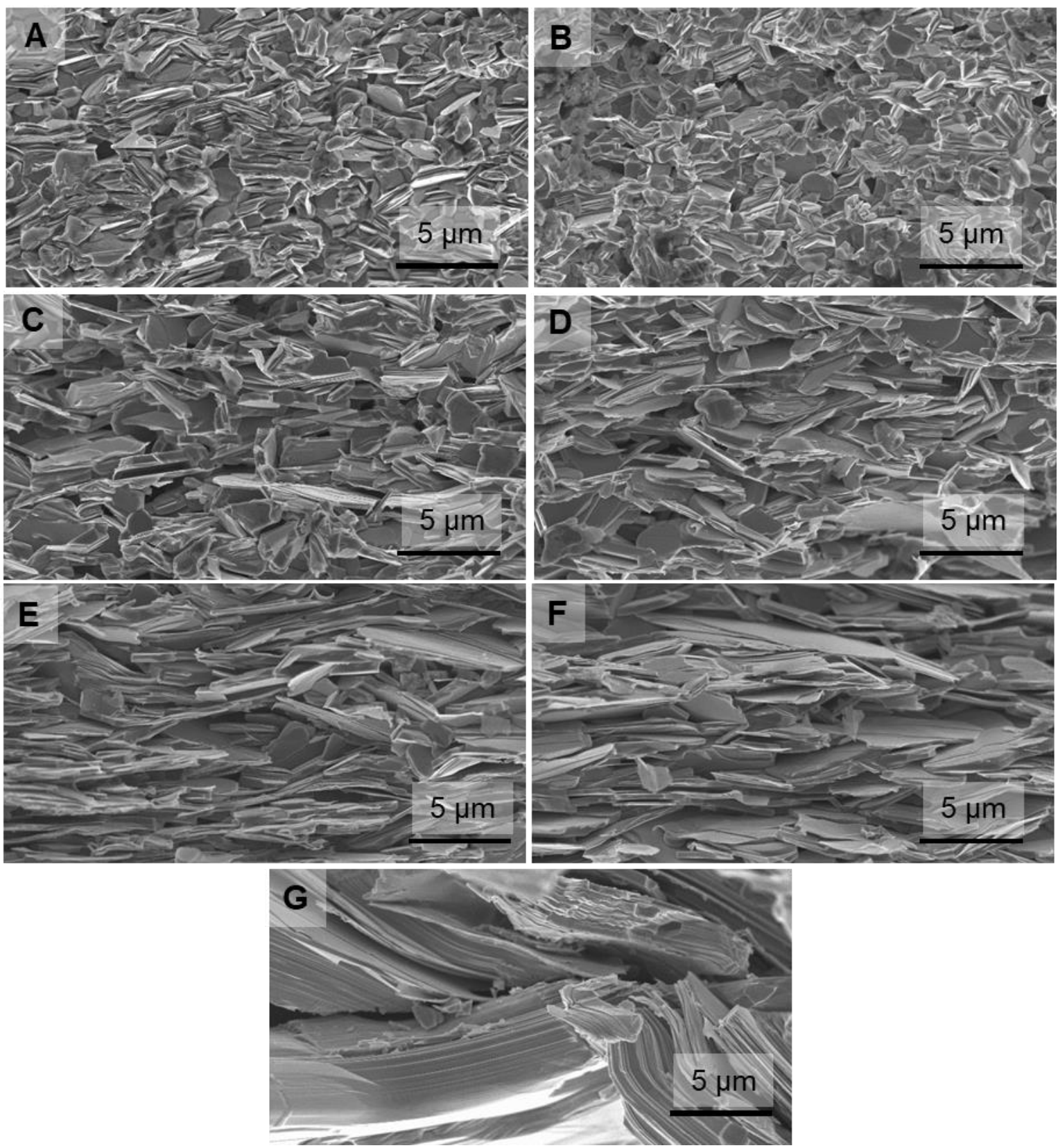

Figure S4: SEM images of $\mathrm{Ca}_{3-x} \mathrm{~Tb}_{x} \mathrm{Co}_{4} \mathrm{O}_{9+\delta} \mathrm{Bl}_{y}$ samples after sintering stage. Crosssectional SEM images from the fractured surface: (A) $\mathrm{Ca}_{3} \mathrm{CO}_{4} \mathrm{O}_{9+\delta}$, (B) $\mathrm{Ca}_{2.95} \mathrm{~Tb}_{0.05} \mathrm{Co}_{4} \mathrm{O}_{9+\delta}$, (C) $\mathrm{Ca}_{2.95} \mathrm{~Tb}_{0.05} \mathrm{Co}_{4} \mathrm{O}_{9+\delta} \mathrm{Bi}_{0.10}$, (D) $\mathrm{Ca}_{2.95} \mathrm{~Tb}_{0.05} \mathrm{Co}_{4} \mathrm{O}_{9+\delta} \mathrm{Bi}_{0.15}$, (E) $\mathrm{Ca}_{2.95} \mathrm{~Tb}_{0.05} \mathrm{Co}_{4} \mathrm{O}_{9+\delta} \mathrm{Bi}_{0.20}$, (F) $\mathrm{Ca}_{2.95} \mathrm{~Tb}_{0.05} \mathrm{CO}_{4} \mathrm{O}_{9+\delta} \mathrm{Bi}_{0.25}$, and (G) $\mathrm{Ca}_{2.95} \mathrm{~Tb}_{0.05} \mathrm{Co}_{4} \mathrm{O}_{9+\delta} \mathrm{Bi}_{0.30}$. 


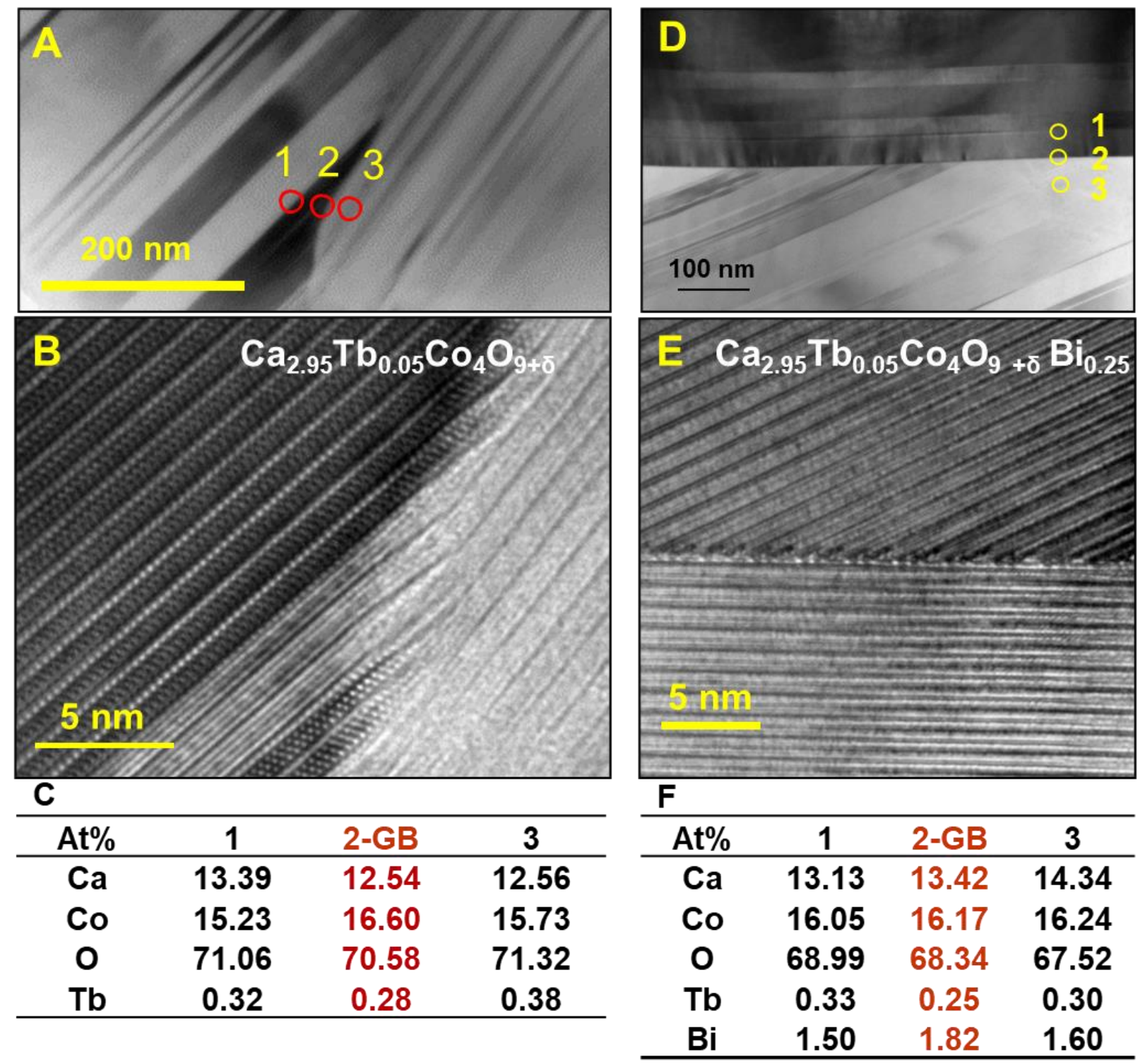

Figure S5: TEM images showing the nanostructure of the $\mathrm{Ca}_{2.95} \mathrm{~Tb}_{0.05} \mathrm{Co}_{4} \mathrm{O}_{9+\delta} \mathrm{Bi}_{y}$ samples. ( $A, B)$ Show the grain boundary from the crystals with different c-axis orientations for $\mathrm{Ca}_{2.95} \mathrm{~Tb}_{0.05} \mathrm{Co}_{4} \mathrm{O}_{9+\delta}$. The red circles on the TEM (A) images indicate the electron beam sample areas for the EDS data acquisition indicated on (C). Show the grain boundary between neighboring crystals with different $c$-axis orientations for $\mathrm{Ca}_{2.95} \mathrm{~Tb}_{0.05} \mathrm{CO}_{4} \mathrm{O}_{9+\delta} \mathrm{Bi}_{0.25}$. The circles on the TEM (D) images indicate the electron beam sample areas for the EDS data acquisition indicated on $(F)$. 
Table S1: Apparent density of the $\mathrm{Ca}_{3-x} \mathrm{~Tb}_{x} \mathrm{Co}_{4} \mathrm{O}_{9+\delta} \mathrm{Bi}_{y}$ samples.

\begin{tabular}{ll}
\hline $\mathrm{Ca}_{3-\mathrm{x}} \mathrm{Tb}_{\mathrm{x}} \mathrm{Co}_{4} \mathrm{O}_{9+\delta} \mathrm{Bi}_{\mathrm{y}}$ & Average Apparent density $\left(\mathrm{g} / \mathrm{cm}^{3}\right)$ \\
$\mathrm{x}=0 \quad \mathrm{y}=0$ & 3.883 \\
$\mathrm{x}=0.05 \mathrm{y}=0$ & 4.182 \\
$\mathrm{x}=0.30 \mathrm{y}=0$ & 4.477 \\
$\mathrm{x}=0.50 \mathrm{y}=0$ & 4.571 \\
$\mathrm{x}=0.05 \mathrm{y}=0.10$ & 3.911 \\
$\mathrm{x}=0.05 \mathrm{y}=0.15$ & 3.909 \\
$\mathrm{x}=0.05 \mathrm{y}=0.20$ & 3.970 \\
$\mathrm{x}=0.05 \mathrm{y}=0.25$ & 4.295 \\
$\mathrm{x}=0.05 \mathrm{y}=0.30$ & 4.701 \\
\hline
\end{tabular}

Table S2: Lattice parameters $a, b_{1}, b_{2}, c$, and $\beta$ for the single doped $\mathrm{Ca}_{3-x} \mathrm{~Tb}_{x} \mathrm{Co}_{4} \mathrm{O}_{9+\delta}(x$ $=0$, and 0.05) and the dual doped $\mathrm{Ca}_{2.95} \mathrm{~Tb}_{0.05} \mathrm{Co}_{4} \mathrm{O}_{9+\delta} B i_{y}(y=0.10,0.15,0.20,0.25$, and 0.30 ) samples. $b_{1}$ and $b_{2}$ refer to $b$-axis lattice parameters in the rock-salt layer $\mathrm{Ca}_{2} \mathrm{CoO}_{3}$ and the $\mathrm{CoO}_{2}$ layer, respectively.

\begin{tabular}{llllcc}
\hline $\mathrm{Ca}_{3-\mathrm{x}} \mathrm{Tb}_{\mathrm{x}} \mathrm{Co}_{4} \mathrm{O}_{9+\delta} \mathrm{Bi}_{\mathrm{v}}$ & $\mathrm{a}(\AA)$ & $\mathrm{b}_{1}(\AA)$ & $\mathrm{b}_{2}(\AA)$ & $\mathrm{c}(\AA)$ & $\beta\left(^{\circ}\right)$ \\
$\mathrm{x}=0 ; \mathrm{y}=0$ & 4.859 & 4.562 & 2.747 & 10.849 & 98.711 \\
$x=0.05 ; \mathrm{y}=0$ & 4.858 & 4.552 & 2.749 & 10.847 & 98.697 \\
$\mathrm{x}=0.05 ; \mathrm{y}=0.10$ & 4.878 & 4.573 & 2.744 & 10.879 & 99.052 \\
$\mathrm{x}=0.05 ; \mathrm{y}=0.15$ & 4.880 & 4.571 & 2.743 & 10.876 & 98.955 \\
$\mathrm{x}=0.05 ; \mathrm{y}=0.20$ & 4.880 & 4.566 & 2.743 & 10.880 & 99.038 \\
$\mathrm{x}=0.05 ; \mathrm{y}=0.25$ & 4.915 & 4.562 & 2.738 & 10.926 & 99.725 \\
$\mathrm{x}=0.05 ; \mathrm{y}=0.30$ & 4.848 & 4.548 & 2.752 & 10.862 & 98.377 \\
\hline
\end{tabular}


Table S3: Progression of the performance of $\mathrm{Ca}_{3} \mathrm{Co}_{4} \mathrm{O}_{9+\delta}$ thermoelectric material.

\begin{tabular}{|c|c|c|c|c|c|c|c|c|}
\hline Ref & Year & Stoichiometry & $\begin{array}{c}S \\
(\mu \mathrm{V} / \mathrm{K}) \\
\end{array}$ & $\begin{array}{c}\rho \\
(\mu \Omega \mathrm{m}) \\
\end{array}$ & $\begin{array}{c}\kappa \\
(\mathrm{W} / \mathrm{Km}) \\
\end{array}$ & $\begin{array}{c}S^{2} / \rho \\
\left(\mathrm{mW} / \mathrm{K}^{2} \mathrm{~m}\right) \\
\end{array}$ & $Z T$ & $\begin{array}{c}T \\
(\mathrm{~K}) \\
\end{array}$ \\
\hline 13 & 2000 & $\mathrm{Ca}_{2.5} \mathrm{Bi}_{0.5} \mathrm{Co}_{4} \mathrm{O}_{9+\delta}$ & 160 & 95 & 1.37 & 0.27 & 0.2 & 973 \\
\hline 67 & 2002 & $\mathrm{Ca}_{2.4} \mathrm{Bi}_{0.3} \mathrm{Na}_{0.3} \mathrm{Co}_{4} \mathrm{O}_{9}$ & 204 & 75 & 1.75 & 0.55 & 0.32 & 1000 \\
\hline 14 & 2003 & $\mathrm{Ca}_{3} \mathrm{Co}_{4} \mathrm{O}_{9}$ single crystal & 245 & 23 & 2.90 & 2.61 & 0.87 & 973 \\
\hline 68 & 2004 & $\mathrm{Ca}_{2.7} \mathrm{Eu}_{0.3} \mathrm{Co}_{4} \mathrm{O}_{9+\delta}$ & 194 & 78 & 1.65 & 0.48 & 0.30 & 1000 \\
\hline 59 & 2004 & $\mathrm{Ca}_{2.7} \mathrm{D}_{\mathrm{y} 0.3} \mathrm{Co}_{4} \mathrm{O}_{9+\delta}$ & 190 & 85 & 1.60 & 0.42 & 0.27 & 1000 \\
\hline 69 & 2005 & $\mathrm{Ca}_{3} \mathrm{Co}_{4} \mathrm{O}_{9} / \mathrm{Ag}-10 \mathrm{wt} \%$ & 175 & 30 & & 1.02 & & 1073 \\
\hline 24 & 2006 & $\mathrm{Ca}_{2.9} \mathrm{Bi}_{0.1} \mathrm{Co}_{4} \mathrm{O}_{9}$ single crystal & 200 & 14.5 & 3.00 & 2.76 & 0.89 & 973 \\
\hline 70 & 2008 & $\mathrm{Ca}_{2.7} \mathrm{Ag}_{0.3} \mathrm{Co}_{4} \mathrm{O}_{9+\delta}$ & 229 & 75 & 2.10 & 0.70 & 0.32 & 1000 \\
\hline 60 & 2008 & $\mathrm{Ca}_{2.7} \mathrm{Gd}_{0.3} \mathrm{Co}_{4} \mathrm{O}_{9+\delta}$ & 182 & 78 & 1.40 & 0.42 & 0.24 & 973 \\
\hline 61 & 2009 & $\mathrm{Ca}_{2.7} \mathrm{Ag}_{0.3} \mathrm{Co}_{4} \mathrm{O}_{9} / \mathrm{Ag}-10 \mathrm{wt} \%$ & 218 & 37 & 2.50 & 1.28 & 0.50 & 1000 \\
\hline 71 & 2009 & $\mathrm{Ca}_{2.7} \mathrm{Y}_{0.3} \mathrm{Co}_{4} \mathrm{O}_{9+\delta}$ & 172 & 85 & 1.55 & 0.35 & 0.22 & 973 \\
\hline 62 & 2010 & $\mathrm{Ca}_{3} \mathrm{Co}_{3.7} \mathrm{Ti}_{0.3} \mathrm{O}_{9}$ & 285 & 165 & 1.80 & 0.49 & 0.29 & 1000 \\
\hline 72 & 2010 & $\mathrm{Ca}_{3} \mathrm{Co}_{3.9} \mathrm{Fe}_{0.1} \mathrm{O}_{9+\delta}$ & 258 & 97 & 1.78 & 0.69 & 0.39 & 1000 \\
\hline 73 & 2010 & $\mathrm{Ca}_{3} \mathrm{Co}_{3.95} \mathrm{Ga}_{0.05} \mathrm{Co}_{9+\delta}$ & 200 & 77 & 1.50 & 0.52 & 0.36 & 1073 \\
\hline 74 & 2011 & $\mathrm{Ca}_{2.97} \mathrm{Ag}_{0.03} \mathrm{Co}_{4} \mathrm{O}_{9+\delta}$ & 200 & 89 & 2.10 & 0.45 & 0.23 & 973 \\
\hline 75 & 2011 & $\mathrm{Ca}_{2.7} \mathrm{Er}_{0.3} \mathrm{Co}_{4} \mathrm{O}_{9+\delta}$ & 192 & 100 & 1.42 & 0.37 & 0.28 & 1073 \\
\hline 76 & 2011 & $\mathrm{Ca}_{2.8} \mathrm{Lu}_{0.2} \mathrm{Co}_{4} \mathrm{O}_{9+\delta}$ & 194 & 95 & 1.20 & 0.40 & 0.36 & 1073 \\
\hline 63 & 2011 & $\mathrm{Ca}_{2.8} \mathrm{Ag}_{0.05} \mathrm{Lu}_{0.15} \mathrm{Co}_{4} \mathrm{O}_{9+\delta}$ & 235 & 72 & 1.40 & 0.77 & 0.61 & 1118 \\
\hline 77 & 2011 & $\mathrm{Ca}_{2.8} \mathrm{Pr}_{0.2} \mathrm{Co}_{4} \mathrm{O}_{9}$ & 201 & 107 & 1.55 & 0.38 & 0.24 & 973 \\
\hline 78 & 2014 & $\mathrm{Ca}_{3} \mathrm{Co}_{3.85} \mathrm{Cr}_{0.15} \mathrm{O}_{9+\delta}$ & 195 & 110 & 2.00 & 0.35 & 0.19 & 1073 \\
\hline 64 & 2014 & $\mathrm{Ca}_{2.7} \mathrm{Bi}_{0.3} \mathrm{Co}_{3.9} \mathrm{Fe}_{0.1} \mathrm{O}_{9+\delta}$ & 182 & 73 & 1.19 & 0.45 & 0.37 & 973 \\
\hline 79 & 2014 & $\mathrm{Ca}_{2.9} \mathrm{La}_{0.1} \mathrm{Co}_{3.9} \mathrm{Fe}_{0.1} \mathrm{O}_{9}$ & 226 & 125 & 1.29 & 0.41 & 0.32 & 1000 \\
\hline 80 & 2014 & $\mathrm{Ca}_{3} \mathrm{Co}_{3.9} \mathrm{Cd}_{0.1} \mathrm{O}_{9}$ & 209 & 82 & 1.50 & 0.53 & 0.35 & 1000 \\
\hline 81 & 2015 & $\mathrm{Ca}_{3} \mathrm{Co}_{4} \mathrm{O}_{9}$ & 132 & 184 & 0.41 & 0.12 & 0.29 & 1000 \\
\hline 34 & 2015 & $\mathrm{Ca}_{3} \mathrm{Ba}_{0.05} \mathrm{Co}_{4} \mathrm{O}_{9+\delta}$ & 185 & 41 & 1.70 & 0.83 & 0.52 & 1073 \\
\hline 82 & 2016 & $\mathrm{Ca}_{2.8} \mathrm{Ba}_{0.1} \mathrm{Pr}_{0.1} \mathrm{Co}_{4} \mathrm{O}_{9+\delta}$ & 207 & 92 & 1.65 & 0.47 & 0.31 & 973 \\
\hline 83 & 2016 & $\mathrm{Ca}_{3} \mathrm{Co}_{4} \mathrm{O}_{9}+5$ wt. $\% \mathrm{~K}_{2} \mathrm{CO}_{3}$ & 230 & 125 & 1.32 & 0.42 & 0.35 & 1073 \\
\hline 25 & 2016 & $\mathrm{Ca}_{2.8} \mathrm{Bi}_{0.2} \mathrm{Co}_{4} \mathrm{O}_{9+\delta}$ & 192 & 50 & 1.90 & 0.74 & 0.43 & 1073 \\
\hline 84 & 2016 & $\mathrm{Ca}_{3} \mathrm{Co}_{4} \mathrm{O}_{9}$ & 170 & 175 & 0.49 & 0.17 & 0.31 & 900 \\
\hline 85 & 2017 & $\mathrm{Ca}_{2.55} \mathrm{Na}_{0.45} \mathrm{Co}_{4} \mathrm{O}_{8.55} \mathrm{~F}_{0.45}$ & 192 & 130 & 2.20 & 0.28 & 0.13 & 873 \\
\hline 65 & 2017 & $\mathrm{Ca}_{3} \mathrm{Co}_{4} \mathrm{O}_{9}$ & 210 & 171 & 0.63 & 0.26 & 0.40 & 1073 \\
\hline 86 & 2017 & $\mathrm{Ca}_{2.9} \mathrm{Bi}_{0.1} \mathrm{Ba}_{0.07} \mathrm{Co}_{4} \mathrm{O}_{9+\delta}$ & 190 & 40 & & 0.90 & & 1073 \\
\hline 33 & 2018 & $\mathrm{Ca}_{3} \mathrm{Co}_{4} \mathrm{O}_{9+\delta} \mathrm{K}_{0.1}$ & 190 & 44 & & 0.82 & & 1073 \\
\hline 87 & 2018 & $\mathrm{Ca}_{2.95} \mathrm{Na}_{0.05} \mathrm{Co}_{3.975} \mathrm{~W}_{0.025} \mathrm{O}_{9}$ & 183 & 130 & 1.26 & 0.26 & 0.21 & 1000 \\
\hline 51 & 2018 & $\mathrm{Ca}_{3} \mathrm{Co}_{4} \mathrm{O}_{9+\delta}$ & 189 & 72 & 1.95 & 0.50 & 0.28 & 1073 \\
\hline 66 & 2019 & $\mathrm{Ca}_{2.25} \mathrm{Na}_{0.3} \mathrm{Bi}_{0.35} \mathrm{~Tb}_{0.1} \mathrm{Co}_{4} \mathrm{O}_{9}$ & 236 & 86 & 1.96 & 0.65 & 0.35 & 1073 \\
\hline 88 & 2019 & $\mathrm{Ca}_{2.97} \mathrm{Sr}_{0.03} \mathrm{Co}_{4} \mathrm{O}_{9}$ & 275 & 65 & 4.40 & 1.16 & 0.29 & 1073 \\
\hline 89 & 2019 & $\mathrm{Ca}_{2.25} \mathrm{Na}_{0.3} \mathrm{Bi}_{0.35} \mathrm{~Tb}_{0.1} \mathrm{Co}_{4} \mathrm{O}_{9}$ & 240 & 70 & & 0.82 & & 1073 \\
\hline$*$ & 2019 & $\mathrm{Ca}_{2.95} \mathrm{~Tb}_{0.05} \mathrm{Co}_{4} \mathrm{O}_{9+\delta} \mathrm{Bi}_{0.25}$ & 212 & 36 & 1.49 & 1.25 & 0.9 & 1073 \\
\hline
\end{tabular}

